\title{
Mechanism of Electrocatalytically Active Precious \\ Metal (Ni, Pd, Pt and Ru) Complexes in Graphene \\ Basal Plane for ORR Application of Novel Fuel Cell
}

Yi Xiao ${ }^{a^{*}}$, Weibin Zhang ${ }^{b^{*}}$

a Institute of Materials Science, TU Darmstadt, 64287 Darmstadt, Germany
b School of Physics and Optoelectronic Engineering, Yangtze University, Jingzhou 434023, PR China
${ }^{*}$ E-mail addresses: yixiao@tmm@tu-darmstadt.de (Y.Xiao); wbzhang@yangtzeu.edu.cn (W. Zhang).

Graphical abstract

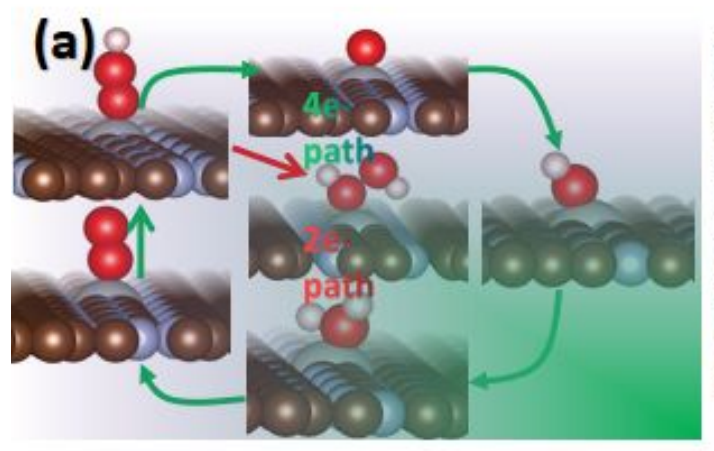

THERMOCHEMISTRY ANALYSIS

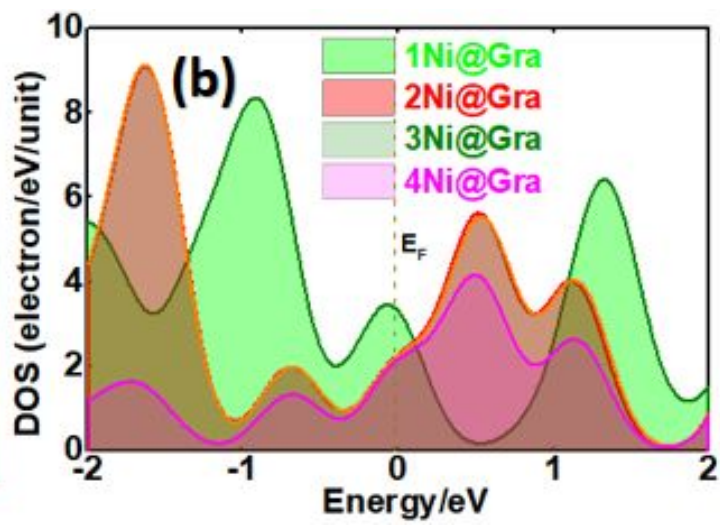

The fundamental thermodynamic equation defined as follow:

$G=H-T S$

Under the standard DFT calculation conditions $(\mathrm{T}=0 \mathrm{~K})$, so the TS is almost negligible. Actually, when the temperatures greater than $0 \mathrm{~K}$ and constant pressure, that means normal temperature and pressure conditions (here $\mathrm{T}=298.15 \mathrm{~K}$ ), zero point energy (ZPE) corrections can be included to finally obtain, enthalpy for a given temperature can be expressed in terms of

$G=H^{0}+\mathrm{ZPE}-\mathrm{T} S$

In addition, the entropy term can be calculated as the sum of the translational, rotational, vibrational, and electronic contributions as to:

$S_{\text {tot }}=S_{\text {trans }}+S_{\text {rot }}+S_{\text {vib }}+S_{\text {ele }}$

and also, the total corrections can be included to finally obtain:

$G=H_{0}+\mathrm{ZPE}-\mathrm{T}\left(S_{t}+S_{r}+S_{v}+S_{e}\right)$

Meanwhile, the enthalpy can be expressed in terms of $\mathrm{H}(0)$ and the heat capacity, $\mathrm{C}_{\mathrm{P}}$ as follow:

$H(T)=H(T)-H(0)=\int_{0}^{T} C_{p} d T$

$\Delta G=\Delta E_{\mathrm{DFT}}+\Delta \mathrm{H}+\Delta \mathrm{ZPE}-T \Delta \mathrm{S}$

(6)

Zero-point energy (ZPE) and entropy corrections (TS)

Since these thermal corrections for the enthalpy have been taken into account for the $\Delta G$ calculation. We shift the chemical potential of the electrons by the equilibrium potential of 1.23 $\mathrm{eV}$ by means of experimental data is proved, where the fuel cell has the maximum potential allowed by thermodynamics, then both electron/proton-transfer steps become uphill, it is therefore likely that one of these is the rate-limiting step. As well known that in the oxygen reduction 
reaction process, the Gibbs free energy change $(\Delta \mathrm{G})$ between two reaction steps involve an electrochemical proton-electron transfer. According to the computational hydrogen electrode (CHE) model, the free energy change $\Delta \mathrm{G}$ is a function of the applied electrode potential at zero-potential $(\mathrm{U}=0 \mathrm{~V})$,

$(1) *+\mathrm{O}_{2}+\left(\mathrm{H}^{+}+\mathrm{e}^{-}\right) \rightarrow * \mathrm{OOH}$

$\Delta G=G\left({ }^{*} \mathrm{OOH}\right)-G\left({ }^{*}\right)-G\left(\mathrm{O}_{2}\right)-G\left(\mathrm{H}^{+}+\mathrm{e}^{-}\right)=$

$G(* \mathrm{OOH})-G(*)-G\left(\mathrm{O}_{2}\right)-G\left[1 / 2 \mathrm{H}_{2}(\mathrm{~g})\right]$

When at equilibrium potential of $\mathrm{U}=1.23 \mathrm{~V}$, taking one proton-electron transfer $\left(\mathrm{H}^{+}+\mathrm{e}^{-}\right)$as follow:

$\Delta G\left({ }^{*} \mathrm{OOH}\right)=G\left({ }^{*} \mathrm{OOH}\right)-G(*)-\mathrm{G}\left(\mathrm{O}_{2}\right)-1 / 2 \mathrm{G}\left(\mathrm{H}_{2}(\mathrm{~g})\right)+\mathrm{e} U$

(2) $* \mathrm{O}_{2}+2\left(\mathrm{H}^{+}+\mathrm{e}^{-}\right) \rightarrow * \mathrm{O}+\mathrm{H}_{2} \mathrm{O}$

$\Delta G\left({ }^{*} \mathrm{O}\right)=G(* \mathrm{O})+\mathrm{G}\left(\mathrm{H}_{2} \mathrm{O}\right)-G(*)-G\left(\mathrm{O}_{2}\right)-2 G\left(\mathrm{H}^{+}+\mathrm{e}^{-}\right)=$

$G(* \mathrm{O})+G\left(\mathrm{H}_{2} \mathrm{O}\right)-G(*)-G\left(\mathrm{O}_{2}\right)-G\left[\mathrm{H}_{2}(\mathrm{~g})\right]$

When at equilibrium potential of $\mathrm{U}=1.23 \mathrm{~V}$, taking two proton-electron transfer $\left(\mathrm{H}^{+}+\mathrm{e}^{-}\right)$as follow:

$\Delta G\left({ }^{*} \mathrm{O}\right)=G\left({ }^{*} \mathrm{O}\right)+G\left(\mathrm{H}_{2} \mathrm{O}\right)-G(*)-G\left(\mathrm{O}_{2}\right)-G\left[\mathrm{H}_{2}(\mathrm{~g})\right]+\mathrm{e} U$

(3) $* \mathrm{O}_{2}+3\left(\mathrm{H}^{+}+\mathrm{e}^{-}\right) \rightarrow * \mathrm{OH}+\mathrm{H}_{2} \mathrm{O}$

$\Delta G(* \mathrm{OH})=G(* \mathrm{OH})+\mathrm{G}\left(\mathrm{H}_{2} \mathrm{O}\right)-G(*)-G\left(\mathrm{O}_{2}\right)-3 G\left(\mathrm{H}^{+}+\mathrm{e}^{-}\right)=$

$G\left({ }^{*} \mathrm{OH}\right)+G\left(\mathrm{H}_{2} \mathrm{O}\right)-G(*)-G\left(\mathrm{O}_{2}\right)-3 / 2 G\left[\mathrm{H}_{2}(\mathrm{~g})\right]$

When at equilibrium potential of $\mathrm{U}=1.23 \mathrm{~V}$, taking three proton-electron transfer $\left(\mathrm{H}^{+}+\mathrm{e}^{-}\right)$as follow:

$\Delta G(* \mathrm{OH})=G(* \mathrm{OH})+G\left(\mathrm{H}_{2} \mathrm{O}\right)-G(*)-G\left(\mathrm{O}_{2}\right)-3 / 2 G\left[\mathrm{H}_{2}(\mathrm{~g})\right]+\mathrm{e} U$,

(4) $* \mathrm{O}_{2}+4\left(\mathrm{H}^{+}+\mathrm{e}^{-}\right) \rightarrow * \mathrm{H}_{2} \mathrm{O}+\mathrm{H}_{2} \mathrm{O}$

$\Delta G\left({ }^{*} \mathrm{H}_{2} \mathrm{O}\right)=G\left({ }^{*} \mathrm{H}_{2} \mathrm{O}\right)+\mathrm{G}\left(\mathrm{H}_{2} \mathrm{O}\right)-G(*)-G\left(\mathrm{O}_{2}\right)-4 G\left(\mathrm{H}^{+}+\mathrm{e}^{-}\right)=$

$G\left({ }^{*} \mathrm{H}_{2} \mathrm{O}\right)+\mathrm{G}\left(\mathrm{H}_{2} \mathrm{O}\right)-G(*)-G\left(\mathrm{O}_{2}\right)-2 G\left[\mathrm{H}_{2}(\mathrm{~g})\right]$

When at equilibrium potential of $\mathrm{U}=1.23 \mathrm{~V}$, taking four proton-electron transfer $\left(\mathrm{H}^{+}+\mathrm{e}^{-}\right)$as follow:

$\Delta G\left({ }^{*} \mathrm{H}_{2} \mathrm{O}\right)=G\left({ }^{*} \mathrm{H}_{2} \mathrm{O}\right)+\mathrm{G}\left(\mathrm{H}_{2} \mathrm{O}\right)-G(*)-G\left(\mathrm{O}_{2}\right)-2 G\left[\mathrm{H}_{2}(\mathrm{~g})\right]+\mathrm{eU}$,

where the asterisk $(*)$ denotes an adsorbed species on doped graphene systems.

During ORR, $\Delta \mathrm{G}$ products refers to the energy of the intermediate species adsorbed on doped graphene systems. We have listed ZPE, TS, and Gibbs free energies of gas molecules and the ORR intermediates in Table S1, Table S2 and Table S3, respectively. Based on the calculated data, the free energy change $\Delta \mathrm{G}$ of each step for the ORR on graphene doping surfaces can be obtained. Meanwhile, For OER to be endothermic reaction nonspontaneous of each elementary step, in order to make it downhill, $\Delta \mathrm{G}>0$. For an idealized ORR mechanism, $\Delta G_{\text {IXn,i }}$ for each step is the result in energy changes will be equal to the formation energy.

$G_{\text {OER }}=\min \left\{\Delta G_{1}, \Delta G_{2}, \Delta G_{3}, \Delta G_{4}\right\}$

$U^{O E R}=\min \left|\frac{\Delta G_{r x n, i}(U=0)}{e v_{e}^{-}}\right|$

$\eta_{\mathrm{OER}}=G_{\mathrm{ORR}} / e-1.23$

\section{Supporting information for Tables and Figures:}

Table S1.Zero Point Energy Corrections and Entropic Contributions to the Free Energies Under the standard conditions, (298.15K, 1 bar). 


\begin{tabular}{ccccc}
\hline Species & $\Delta \mathrm{E}_{\text {ZPE }}(\mathrm{eV})$ & $\Delta \mathrm{U}(\mathrm{T})(\mathrm{eV})$ & $\Delta \mathrm{H}(\mathrm{T})(\mathrm{eV})$ & $\Delta \mathrm{G}(\mathrm{T})(\mathrm{eV})$ \\
\hline $\mathrm{O}_{2}$ & 0.096632 & 0.160967 & 0.186659 & -0.447249 \\
$\mathrm{H}_{2} \mathrm{O}$ & 0.572122 & 0.649304 & 0.674996 & 0.050668 \\
$\mathrm{H}_{2}$ & 0.260748 & 0.324978 & 0.35067 & -0.080206 \\
$\mathrm{H}_{2} \mathrm{O}_{2}$ & 0.685612 & 0.786121 & 0.811813 & 0.019156 \\
\hline
\end{tabular}

Table S2. Gibbs free energies (G) for correction data, zero-point energies (ZPE), entropies multiplied by $\mathrm{T}$ (= $298.15 \mathrm{~K})(\mathrm{TS})$, and relative free energies $(\Delta \mathrm{G}$, at $1.23 \mathrm{~V})$ of ORR intermediates used in the main text. Path I (I 1 and I 2 ) and II denote four-electron and two-electron reduction pathways respectively, as demonstrated in Figure 1.Table S5. Zero-point energy (ZPE, in eV) and entropy corrections (TS, in eV) at T $=298 \mathrm{~K}$ for adsorbates.

\begin{tabular}{|c|c|c|c|c|c|c|c|c|c|}
\hline $1 \mathrm{Ni}$ & $T S$ & $\boldsymbol{H}$ & $E_{Z P E}$ & $H+E_{Z P E^{-}}-T S$ & $2 \mathrm{Ni}$ & $T S$ & $\boldsymbol{H}$ & $E_{Z P E}$ & $H+E_{Z P E}-T S$ \\
\hline$* \mathrm{O}_{2}$ & 0.5272 & 0.2870 & 2.6536 & 2.4134 & $* \mathrm{O}_{2}$ & 0.4710 & 0.2494 & 2.2211 & 1.9995 \\
\hline$* \mathrm{OOH}$ & 0.5288 & 0.2987 & 2.9692 & 2.7390 & $* \mathrm{OOH}$ & 0.4759 & 0.2648 & 2.4337 & 2.2226 \\
\hline$* \mathrm{O}$ & 0.4409 & 0.2474 & 2.4739 & 2.2804 & $* \mathrm{O}$ & 0.3544 & 0.1928 & 2.0269 & 1.8652 \\
\hline$* \mathrm{OH}$ & 0.4603 & 0.2688 & 2.8611 & 2.6695 & $* \mathrm{OH}$ & 0.4225 & 0.2398 & 2.2990 & 2.1163 \\
\hline$* \mathrm{H}_{2} \mathrm{O}$ & 0.5171 & 0.2800 & 3.1360 & 2.8989 & $* \mathrm{H}_{2} \mathrm{O}$ & 0.4246 & 0.2372 & 2.7280 & 2.5406 \\
\hline$* \mathrm{H}_{2} \mathrm{O}_{2}$ & 0.5664 & 0.3074 & 3.2737 & 3.0147 & $* \mathrm{H}_{2} \mathrm{O}_{2}$ & 0.4860 & 0.2599 & 2.8439 & 2.6177 \\
\hline $2 * \mathrm{O}$ & 0.3982 & 0.2432 & 2.6733 & 2.5183 & $2 * \mathrm{O}$ & 0.4352 & 0.2363 & 2.1155 & 1.9166 \\
\hline$* \mathrm{OHO}$ & 0.4949 & 0.2891 & 2.9431 & 2.7373 & $* \mathrm{OHO}$ & 0.4372 & 0.2595 & 2.5389 & 2.3612 \\
\hline$* \mathrm{H}$ & 0.3433 & 0.2187 & 2.6721 & 2.5475 & $* \mathrm{H}$ & 0.3700 & 0.2166 & 2.1252 & 1.9718 \\
\hline $3 \mathrm{Ni}$ & $T S$ & $\boldsymbol{H}$ & $E_{Z P E}$ & $H+E_{Z P E^{-}}-T S$ & $4 \mathrm{Ni}$ & $T S$ & $\boldsymbol{H}$ & $E_{Z P E}$ & $H+E_{Z P E^{-}} T S$ \\
\hline$* \mathrm{O}_{2}$ & 0.4738 & 0.2513 & 2.2174 & 1.9949 & $* \mathrm{O}_{2}$ & 0.5977 & 0.3343 & 3.6602 & 3.3967 \\
\hline$* \mathrm{OOH}$ & 0.4836 & 0.2650 & 2.4336 & 2.2150 & $* \mathrm{OOH}$ & 0.6645 & 0.3743 & 3.9674 & 3.6772 \\
\hline$* \mathrm{O}$ & 0.3508 & 0.1925 & 2.0250 & 1.8667 & $* \mathrm{O}$ & 0.5306 & 0.2987 & 3.4681 & 3.2362 \\
\hline$* \mathrm{OH}$ & 0.4667 & 0.2558 & 2.3154 & 2.1046 & $* \mathrm{OH}$ & 0.5453 & 0.3215 & 3.8479 & 3.6241 \\
\hline$* \mathrm{H}_{2} \mathrm{O}$ & 0.4900 & 0.2613 & 2.7287 & 2.4999 & $* \mathrm{H}_{2} \mathrm{O}$ & 0.5973 & 0.3421 & 4.1641 & 3.9089 \\
\hline$* \mathrm{H}_{2} \mathrm{O}_{2}$ & 0.5389 & 0.2840 & 2.8433 & 2.5884 & $* \mathrm{H}_{2} \mathrm{O}_{2}$ & 0.7058 & 0.3859 & 4.2783 & 3.9585 \\
\hline $2 * \mathrm{O}$ & 0.3781 & 0.2316 & 2.2819 & 2.1354 & $2 * \mathrm{O}$ & 0.6538 & 0.3585 & 3.5734 & 3.2782 \\
\hline$* \mathrm{OHO}$ & 0.4789 & 0.2682 & 2.5135 & 2.3028 & $* \mathrm{OHO}$ & 0.6614 & 0.3708 & 3.8109 & 3.5202 \\
\hline$*^{H} \mathrm{H}$ & 0.3168 & 0.1939 & 2.1221 & 1.9992 & ${ }^{*} \mathrm{H}$ & 0.4660 & 0.2898 & 3.6738 & 3.4977 \\
\hline 1Pd & $T S$ & $\boldsymbol{H}$ & $E_{Z P E}$ & $H+E_{Z P E}-T S$ & 2Pd & $T S$ & $\boldsymbol{H}$ & $E_{Z P E}$ & $H+E_{Z P E^{-}}-T S$ \\
\hline$* \mathrm{O}_{2}$ & 0.4953 & 0.2680 & 2.7147 & 2.4873 & $* \mathrm{O}_{2}$ & 0.4568 & 0.2413 & 2.2903 & 2.0747 \\
\hline$* \mathrm{OOH}$ & 0.6267 & 0.3281 & 3.0012 & 2.7026 & $* \mathrm{OOH}$ & 0.5536 & 0.2861 & 2.5651 & 2.2977 \\
\hline$* \mathrm{O}$ & 0.4033 & 0.2439 & 2.6216 & 2.4623 & $* \mathrm{O}$ & 0.4365 & 0.2419 & 2.1804 & 1.9858 \\
\hline$* \mathrm{OH}$ & 0.4821 & 0.2735 & 2.9189 & 2.7103 & $* \mathrm{OH}$ & 0.4324 & 0.2453 & 2.5043 & 2.3172 \\
\hline$* \mathrm{H}_{2} \mathrm{O}$ & 0.5803 & 0.3064 & 3.2009 & 2.9270 & ${ }^{*} \mathrm{H}_{2} \mathrm{O}$ & 0.5521 & 0.2929 & 2.7907 & 2.5315 \\
\hline$* \mathrm{H}_{2} \mathrm{O}_{2}$ & 0.5807 & 0.3089 & 3.3371 & 3.0652 & $* \mathrm{H}_{2} \mathrm{O}_{2}$ & 0.5489 & 0.2865 & 2.9056 & 2.6433 \\
\hline $2 * \mathrm{O}$ & 0.4587 & 0.2697 & 2.7102 & 2.5212 & $2 * \mathrm{O}$ & 0.4005 & 0.2374 & 2.2548 & 2.0917 \\
\hline$* \mathrm{OHO}$ & 0.4941 & 0.2912 & 2.9419 & 2.7391 & $* \mathrm{OHO}$ & 0.4799 & 0.2779 & 2.5823 & 2.3803 \\
\hline$* \mathrm{H}$ & 0.3401 & 0.2134 & 2.7039 & 2.5772 & $* \mathrm{H}$ & 0.3945 & 0.2203 & 2.2066 & 2.0324 \\
\hline 3Pd & $T S$ & $\boldsymbol{H}$ & $E_{Z P E}$ & $H+E_{Z P E}-T S$ & 4Pd & $T S$ & $\boldsymbol{H}$ & $E_{Z P E}$ & $H+E_{Z P E}-T S$ \\
\hline$* \mathrm{O}_{2}$ & 0.4600 & 0.2424 & 2.2869 & 2.0693 & $* \mathrm{O}_{2}$ & 0.6359 & 0.3424 & 3.6932 & 3.3997 \\
\hline
\end{tabular}




\begin{tabular}{|c|c|c|c|c|c|c|c|c|c|}
\hline$* \mathrm{OOH}$ & 0.5504 & 0.2862 & 2.5646 & 2.3003 & $* \mathrm{OOH}$ & 0.6574 & 0.3634 & 4.0005 & 3.7066 \\
\hline$* \mathrm{O}$ & 0.4300 & 0.2354 & 2.2010 & 2.0063 & $* \mathrm{O}$ & 0.4926 & 0.2993 & 3.6282 & 3.4350 \\
\hline$* \mathrm{OH}$ & 0.4324 & 0.2445 & 2.5014 & 2.3134 & $* \mathrm{OH}$ & 0.5306 & 0.3106 & 3.8748 & 3.6548 \\
\hline$* \mathrm{H}_{2} \mathrm{O}$ & 0.4905 & 0.2616 & 2.7626 & 2.5337 & $* \mathrm{H}_{2} \mathrm{O}$ & 0.6921 & 0.3720 & 4.2020 & 3.8819 \\
\hline$* \mathrm{H}_{2} \mathrm{O}_{2}$ & 0.5997 & 0.3092 & 2.9019 & 2.6114 & $* \mathrm{H}_{2} \mathrm{O}_{2}$ & 0.7074 & 0.3807 & 4.3277 & 4.0010 \\
\hline $2 * \mathrm{O}$ & 0.4450 & 0.2558 & 2.2521 & 2.0629 & $2 * \mathrm{O}$ & 0.5337 & 0.3210 & 3.7375 & 3.5248 \\
\hline$* \mathrm{OHO}$ & 0.4379 & 0.2573 & 2.5907 & 2.4101 & $* \mathrm{OHO}$ & 0.5661 & 0.3375 & 4.0561 & 3.8276 \\
\hline${ }^{*} \mathrm{H}$ & 0.3375 & 0.1985 & 2.2011 & 2.0621 & $*^{\mathrm{H}}$ & 0.4943 & 0.2963 & 3.6999 & 3.5019 \\
\hline $1 \mathrm{Pt}$ & $T S$ & $\boldsymbol{H}$ & $E_{Z P E}$ & $H+E_{Z P E}-T S$ & $2 \mathrm{Pt}$ & $T S$ & $\boldsymbol{H}$ & $E_{Z P E}$ & $H+E_{Z P E^{-}} T S$ \\
\hline$* \mathrm{O}_{2}$ & 0.4945 & 0.2662 & 2.7186 & 2.4903 & $* \mathrm{O}_{2}$ & 0.4825 & 0.2552 & 2.3143 & 2.0870 \\
\hline$* \mathrm{OOH}$ & 0.5597 & 0.2995 & 3.0082 & 2.7480 & $* \mathrm{OOH}$ & 0.5723 & 0.2965 & 2.6023 & 2.3265 \\
\hline$* \mathrm{O}$ & 0.3886 & 0.2364 & 2.6572 & 2.5051 & $* \mathrm{O}$ & 0.4051 & 0.2288 & 2.2139 & 2.0375 \\
\hline$* \mathrm{OH}$ & 0.4821 & 0.2712 & 2.9254 & 2.7145 & $* \mathrm{OH}$ & 0.4288 & 0.2422 & 2.5061 & 2.3196 \\
\hline$* \mathrm{H}_{2} \mathrm{O}$ & 0.5118 & 0.2810 & 3.2029 & 2.9721 & $* \mathrm{H}_{2} \mathrm{O}$ & 0.5101 & 0.2740 & 2.7968 & 2.5607 \\
\hline$* \mathrm{H}_{2} \mathrm{O}_{2}$ & 0.5615 & 0.3058 & 3.3401 & 3.0844 & $* \mathrm{H}_{2} \mathrm{O}_{2}$ & 0.5228 & 0.2786 & 2.9291 & 2.6849 \\
\hline $2 * \mathrm{O}$ & 0.4276 & 0.2598 & 2.7079 & 2.5401 & $2 * \mathrm{O}$ & 0.4697 & 0.2688 & 2.2913 & 2.0904 \\
\hline$* \mathrm{OHO}$ & 0.4653 & 0.2808 & 2.9937 & 2.8092 & $* \mathrm{OHO}$ & 0.4816 & 0.2719 & 2.6539 & 2.4442 \\
\hline$* \mathrm{H}$ & 0.3998 & 0.2345 & 2.7142 & 2.5489 & ${ }^{*} \mathrm{H}$ & 0.3232 & 0.1905 & 2.2031 & 2.0704 \\
\hline $3 P t$ & $T S$ & $\boldsymbol{H}$ & $E_{Z P E}$ & $H+E_{Z P E^{-}}-T S$ & $4 \mathrm{Pt}$ & $T S$ & $\boldsymbol{H}$ & $E_{Z P E}$ & $H+E_{Z P E^{-}}-T S$ \\
\hline$* \mathrm{O}_{2}$ & 0.4527 & 0.2407 & 2.3052 & 2.0932 & $* \mathrm{O}_{2}$ & 0.6342 & 0.3406 & 3.7458 & 3.4522 \\
\hline$* \mathrm{OOH}$ & 0.4792 & 0.2559 & 2.5871 & 2.3637 & $* \mathrm{OOH}$ & 0.5721 & 0.3253 & 3.8532 & 3.6064 \\
\hline$* \mathrm{O}$ & 0.4573 & 0.2426 & 1.8527 & 1.6381 & $* \mathrm{O}$ & 0.5461 & 0.3175 & 3.6634 & 3.4348 \\
\hline$* \mathrm{OH}$ & 0.4198 & 0.2395 & 2.5136 & 2.3332 & $* \mathrm{OH}$ & 0.5136 & 0.3074 & 3.8887 & 3.6825 \\
\hline$* \mathrm{H}_{2} \mathrm{O}$ & 0.5412 & 0.2809 & 2.7857 & 2.5255 & $* \mathrm{H}_{2} \mathrm{O}$ & 0.6519 & 0.3514 & 4.2372 & 3.9367 \\
\hline$* \mathrm{H}_{2} \mathrm{O}_{2}$ & 0.5547 & 0.2974 & 2.9297 & 2.6723 & $* \mathrm{H}_{2} \mathrm{O}_{2}$ & 0.7103 & 0.3798 & 4.3692 & 4.0387 \\
\hline $2 * \mathrm{O}$ & 0.4376 & 0.2532 & 2.2380 & 2.0536 & $2 * \mathrm{O}$ & 0.5196 & 0.3148 & 3.6996 & 3.4948 \\
\hline$* \mathrm{OHO}$ & 0.4248 & 0.2535 & 2.5894 & 2.4181 & $* \mathrm{OHO}$ & 0.5555 & 0.3301 & 4.0758 & 3.8504 \\
\hline${ }^{*} \mathrm{H}$ & 0.2629 & 0.1668 & 2.1979 & 2.1017 & ${ }^{*} \mathrm{H}$ & 0.4820 & 0.2886 & 3.7100 & 3.5165 \\
\hline $1 \mathrm{Ru}$ & $T S$ & $H$ & $E_{Z P E}$ & $H+E_{Z P E^{-}} T S$ & $2 \mathrm{Ru}$ & TS & $H$ & $E_{Z P E}$ & $H+E_{Z P E^{-}} T S$ \\
\hline$* \mathrm{O}_{2}$ & 0.4683 & 0.2639 & 2.6792 & 2.4748 & $* \mathrm{O}_{2}$ & 0.4759 & 0.2614 & 2.2865 & 2.0720 \\
\hline$* \mathrm{OOH}$ & 0.5022 & 0.2805 & 2.9247 & 2.7030 & $* \mathrm{OOH}$ & 0.5205 & 0.2794 & 2.5694 & 2.3283 \\
\hline$* \mathrm{O}$ & 0.3730 & 0.2299 & 2.6464 & 2.5033 & $* \mathrm{O}$ & 0.3268 & 0.1935 & 2.1248 & 1.9915 \\
\hline$* \mathrm{OH}$ & 0.4021 & 0.2372 & 2.9319 & 2.7670 & $* \mathrm{OH}$ & 0.4444 & 0.2437 & 2.4872 & 2.2865 \\
\hline$* \mathrm{H}_{2} \mathrm{O}$ & 0.4650 & 0.2599 & 3.2003 & 2.9952 & $* \mathrm{H}_{2} \mathrm{O}$ & 0.5317 & 0.2771 & 2.6723 & 2.4178 \\
\hline$* \mathrm{H}_{2} \mathrm{O}_{2}$ & 0.6506 & 0.3226 & 3.2769 & 2.9489 & ${ }^{*} \mathrm{H}_{2} \mathrm{O}_{2}$ & 0.4541 & 0.2467 & 2.7928 & 2.5854 \\
\hline $2 * \mathrm{O}$ & 0.4579 & 0.2717 & 2.6920 & 2.5058 & $2 * \mathrm{O}$ & 0.4211 & 0.2459 & 2.2450 & 2.0698 \\
\hline$* \mathrm{OHO}$ & 0.4826 & 0.2856 & 2.9396 & 2.7425 & $* \mathrm{OHO}$ & 0.4731 & 0.2697 & 2.3995 & 2.1960 \\
\hline${ }^{*} \mathrm{H}$ & 0.3520 & 0.2131 & 2.7951 & 2.6562 & ${ }^{*} \mathrm{H}$ & 0.3701 & 0.2080 & 2.4026 & 2.2405 \\
\hline $3 R \mathbf{u}$ & $T S$ & $\boldsymbol{H}$ & $E_{Z P E}$ & $H+E_{Z P E^{-}} T S$ & $4 \mathrm{Ru}$ & $T S$ & $\boldsymbol{H}$ & $E_{Z P E}$ & $H+E_{Z P E}-T S$ \\
\hline$* \mathrm{O}_{2}$ & 0.4820 & 0.2629 & 2.2812 & 2.0621 & $* \mathrm{O}_{2}$ & 0.5886 & 0.3367 & 3.6719 & 3.4200 \\
\hline$* \mathrm{OOH}$ & 0.5977 & 0.3107 & 2.5602 & 2.2732 & $* \mathrm{OOH}$ & 0.5664 & 0.3301 & 3.9209 & 3.6846 \\
\hline${ }^{*} \mathrm{O}$ & 0.3225 & 0.1925 & 2.1262 & 1.9962 & $* \mathrm{O}$ & 0.4957 & 0.3033 & 3.6436 & 3.4512 \\
\hline
\end{tabular}




\begin{tabular}{|c|c|c|c|c|c|c|c|c|c|}
\hline$* \mathrm{OH}$ & 0.4472 & 0.2437 & 2.4849 & 2.2814 & $* \mathrm{OH}$ & 0.5294 & 0.3110 & 3.9273 & 3.7088 \\
\hline$* \mathrm{H}_{2} \mathrm{O}$ & 0.5370 & 0.2789 & 2.6674 & 2.4093 & $* \mathrm{H}_{2} \mathrm{O}$ & 0.5601 & 0.3230 & 3.9448 & 3.7078 \\
\hline$* \mathrm{H}_{2} \mathrm{O}_{2}$ & 0.4853 & 0.2573 & 2.8291 & 2.6011 & $* \mathrm{H}_{2} \mathrm{O}_{2}$ & 0.6756 & 0.3608 & 4.3072 & 3.9923 \\
\hline $2 * \mathrm{O}$ & 0.3422 & 0.2180 & 2.3202 & 2.1959 & $2 * \mathrm{O}$ & 0.6211 & 0.3572 & 3.5913 & 3.3274 \\
\hline$* \mathrm{OHO}$ & 0.4762 & 0.2691 & 2.4104 & 2.2033 & $* \mathrm{OHO}$ & 0.5340 & 0.3258 & 3.9945 & 3.7863 \\
\hline${ }^{*} \mathrm{H}$ & 0.3706 & 0.2091 & 2.4005 & 2.2390 & ${ }^{*} \mathrm{H}$ & 0.4761 & 0.2846 & 3.8162 & 3.6247 \\
\hline
\end{tabular}
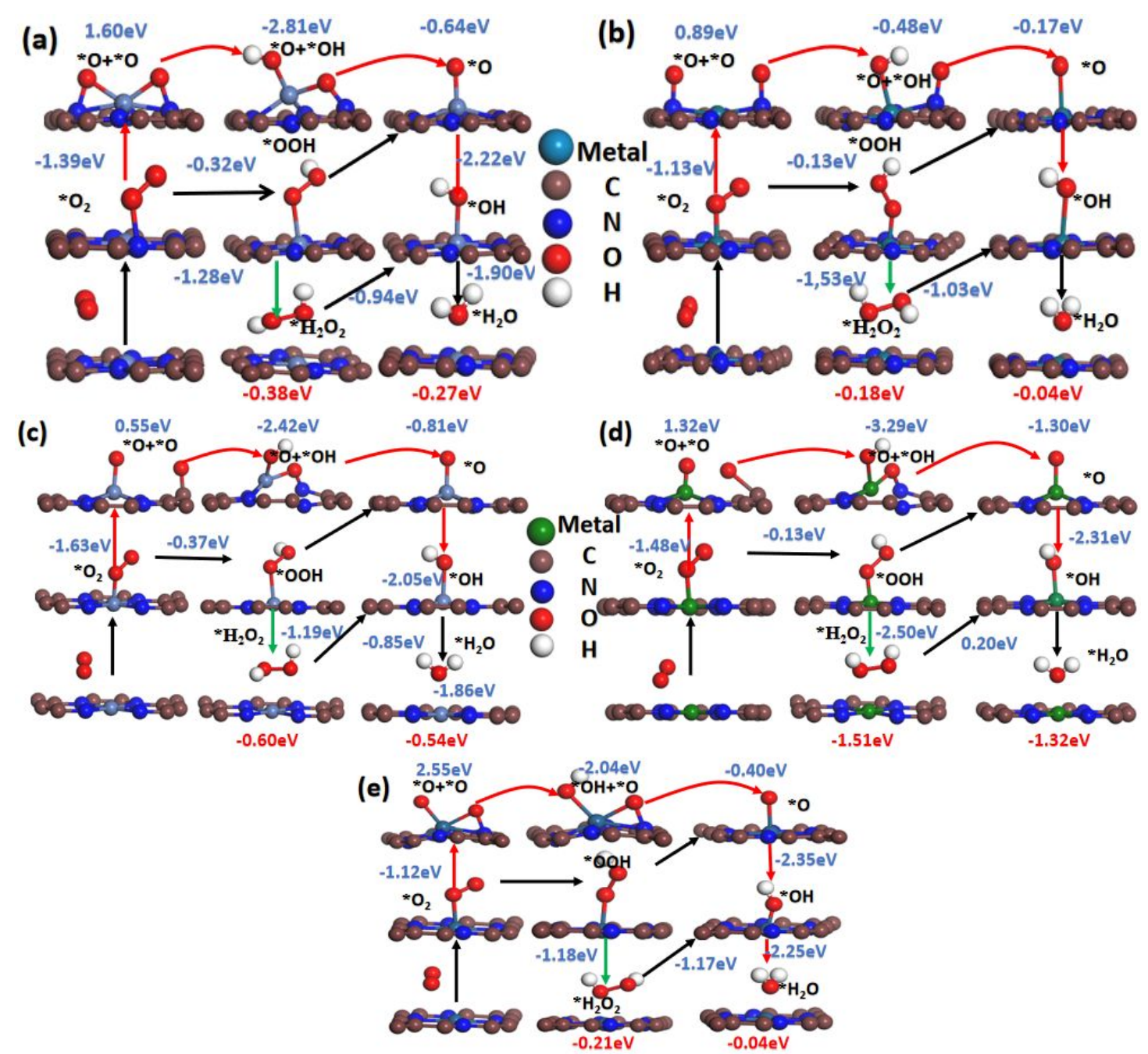

Figure $\mathrm{S} 1$ The local atomic structures ( electrocatalytically active center) of computational models of adsorption structures of the ORR $\left(\mathrm{O}_{2}, \mathrm{OOH}, \mathrm{O}, \mathrm{OH}, \mathrm{H}_{2} \mathrm{O}, 2 \mathrm{O}, \mathrm{OH}+\mathrm{O}\right.$ and $\left.\mathrm{H}_{2} \mathrm{O}_{2}\right)$ adsorbates systems on a single metal-doped graphene monolayer: (a)1Ni@Gra, (b)2Ni@Gra, (c)3Ni@Gra, (d)4Ni@Gra. 


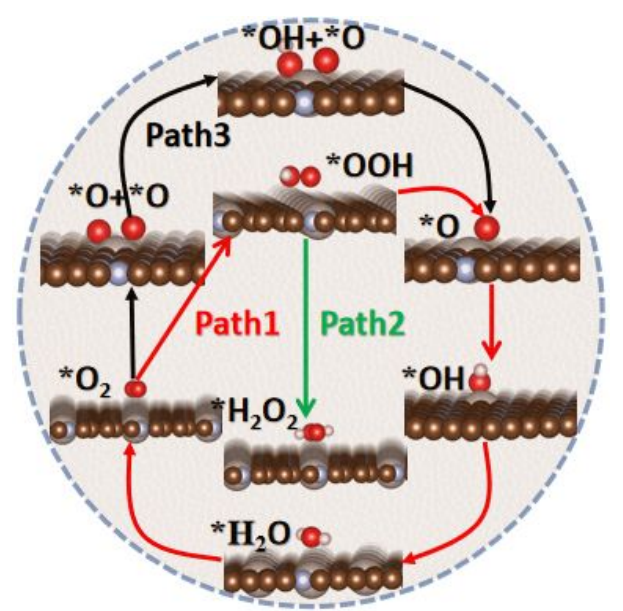

Figure.S2 The entire schematic of ORR pathways on a Metal-N co-doped graphene.
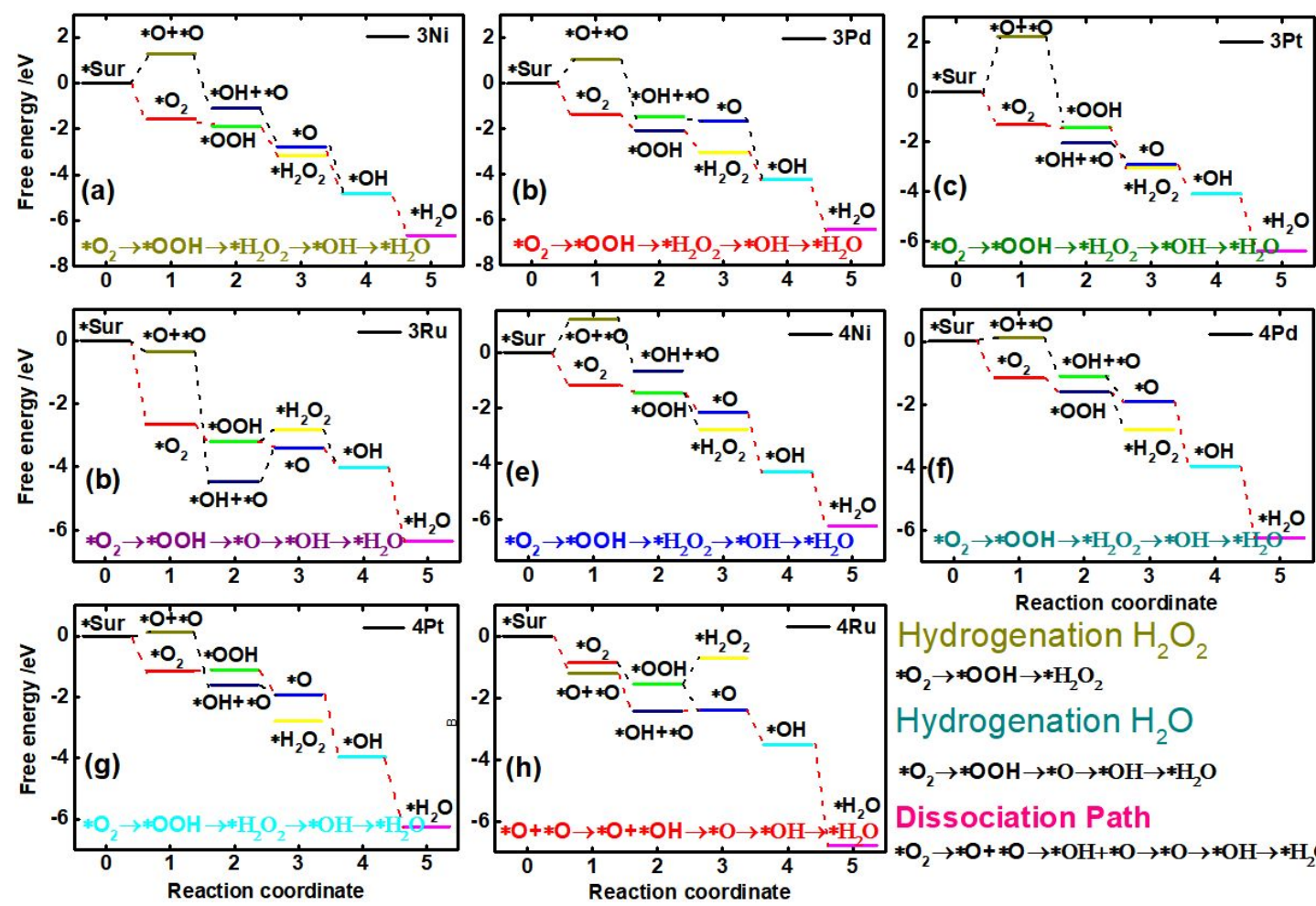

Hydrogenation $\mathrm{H}_{2} \mathrm{O}_{2}$ $* \mathrm{O}_{2} \rightarrow * \mathrm{OOH} \rightarrow * \mathrm{H}_{2} \mathrm{O}_{2}$ Hydrogenation $\mathrm{H}_{2} \mathrm{O}$ $* \mathrm{O}_{2} \rightarrow * \mathrm{OOH} \rightarrow * \mathrm{O} \rightarrow * \mathrm{OH} \rightarrow * \mathrm{H}_{2} \mathrm{O}$ Dissociation Path

Figure S3. DFT study of the ORR on 2D Double Metal Sulfide $\left(\mathrm{M}_{2} \mathrm{~S}_{2}\right)$ surface in acid solutions. Reaction free energy diagrams for the 2e- (red) and 4e-ORR (blue) pathways at the hydrogen reversible potential and surface proton transfers. The most stable adsorption configurations of ORR intermediates chemical species adsorbed in (a) $\mathrm{Sc}_{2} \mathrm{~S}_{2}$ (b) $\mathrm{Ti}_{2} \mathrm{~S}_{2}$ (c) $\mathrm{V}_{2} \mathrm{~S}_{2}$ (d) $\mathrm{Cr}_{2} \mathrm{~S}_{2}$ (e) $\mathrm{Mn}_{2} \mathrm{~S}_{2}$ (f) $\mathrm{Co}_{2} \mathrm{~S}_{2}$ (g) $\mathrm{Ni}_{2} \mathrm{~S}_{2}$ (h) $\mathrm{Zr}_{2} \mathrm{~S}_{2}$ (i) $\mathrm{Nb}_{2} \mathrm{~S}_{2}$ (j) $\mathrm{Mo}_{2} \mathrm{~S}_{2}$ (k) $\mathrm{Rh}_{2} \mathrm{~S}_{2}$ (1) $\mathrm{Hf}_{2} \mathrm{~S}_{2}$ (m) $\mathrm{Ta}_{2} \mathrm{~S}_{2}$ (n) $\mathrm{W}_{2} \mathrm{~S}_{2}$ (o) $\mathrm{Ir}_{2} \mathrm{~S}_{2}$. In the figure, the gray, blue, red, and white balls represent $\mathrm{M}, \mathrm{S}, \mathrm{O}$ and $\mathrm{H}$ atoms, respectively. 

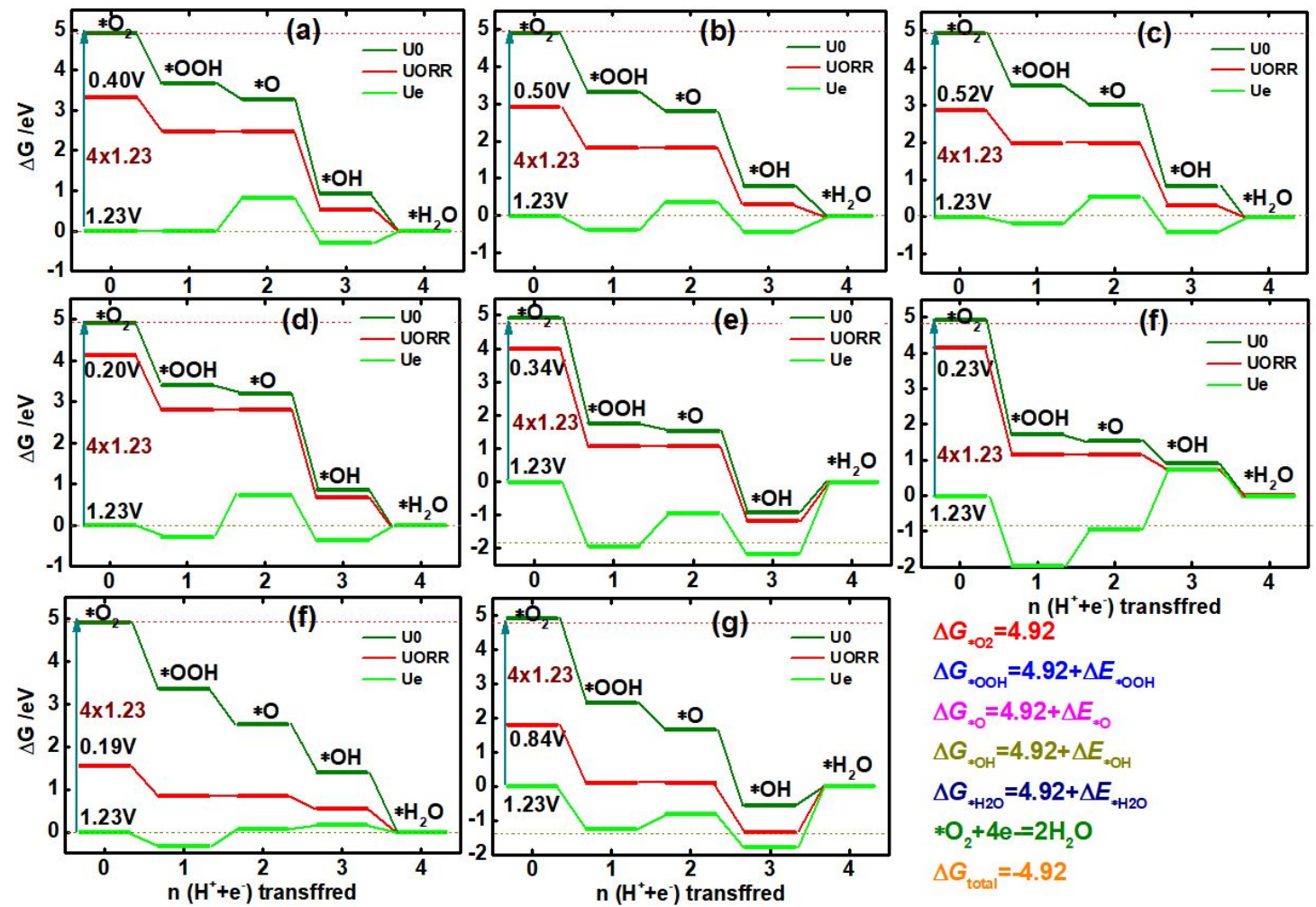

$\Delta G_{* 02}=4.92$

$\Delta G_{* \mathrm{OOH}}=4.92+\Delta E_{\text {×OOH }}$

$\Delta G_{* 0}=4.92+\Delta E_{* 0}$

$\Delta G_{* \mathrm{OH}}=4.92+\Delta E_{* \mathrm{OH}}$

$\Delta G_{*+120}=4.92+\Delta E_{*+12 O}$

$* \mathrm{O}_{2}+4 \mathrm{e}=2 \mathrm{H}_{2} \mathrm{O}$

$\Delta G_{\text {total }}=-4.92$

Figure.S4 The possible ORR and OER pathways on single copper doped graphite, the free energy profiles for the ORR pathway (a-b) and the OER pathway (c-d) on $\mathrm{CuN}_{4}$-graphite surface for the complete $\mathrm{O}_{2}$ reduction/oxidation at different potentials.

Table S3. Total Gibbs free energies at $298.15 \mathrm{~K}$, in eV, corresponding to the isolated $\mathrm{O}_{2}, \mathrm{OOH}, \mathrm{O}, \mathrm{OH}, \mathrm{H}_{2} \mathrm{O}_{2}, 2 \mathrm{O}$, OHO, $\mathrm{H}_{2}$, and $\mathrm{H}_{2} \mathrm{O}$ species, along the $\mathrm{O}_{2}$ conversion into $\mathrm{H}_{2} \mathrm{O}$ or $\mathrm{H}_{2} \mathrm{O}_{2}$ mechanism catalysed by different types group $\mathrm{M} @ \mathrm{Gra}(\mathrm{M}=\mathrm{Ni}, \mathrm{Pd}, \mathrm{Pt}$, and $\mathrm{Ru})$.

\begin{tabular}{cccccccccccc}
\hline $\mathrm{Sys}$ & $\boldsymbol{G}_{\text {DFT }}$ & $\boldsymbol{G}_{\text {tot }}$ & $\mathrm{Sys}$ & $\boldsymbol{G}_{\text {DFT }}$ & $\boldsymbol{G}_{\text {tot }}$ & $\mathrm{Sys}$ & $\boldsymbol{G}_{\text {DFT }}$ & $\boldsymbol{G}_{\text {tot }}$ & $\mathrm{Sys}$ & $\boldsymbol{G}_{\text {DFT }}$ & $\boldsymbol{G}_{\text {tot }}$ \\
\hline $1 \mathrm{Ni}$ & -147.99 & -170.23 & $1 \mathrm{Pd}$ & -147.28 & -169.53 & $1 \mathrm{Pt}$ & -148.26 & -170.51 & $1 \mathrm{Ru}$ & -149.47 & -171.72 \\
\hline $\mathrm{O}_{2}$ & -158.10 & -171.62 & $\mathrm{O}_{2}$ & -157.14 & -170.66 & $\mathrm{O}_{2}$ & -158.12 & -171.63 & $\mathrm{O}_{2}$ & -160.98 & -174.50 \\
$\mathrm{OOH}$ & -161.80 & -171.94 & $\mathrm{OOH}$ & -160.64 & -170.78 & $\mathrm{OOH}$ & -161.61 & -171.75 & $\mathrm{OOH}$ & -165.06 & -175.20 \\
$\mathrm{O}$ & -151.60 & -172.58 & $\mathrm{O}$ & -149.97 & -170.95 & $\mathrm{O}$ & -151.17 & -172.15 & $\mathrm{O}$ & -154.29 & -175.27 \\
$\mathrm{OH}$ & -157.20 & -174.80 & $\mathrm{OH}$ & -155.91 & -173.51 & $\mathrm{OH}$ & -156.90 & -174.50 & $\mathrm{OH}$ & -159.59 & -177.19 \\
$\mathrm{H}_{2} \mathrm{O}$ & -162.48 & -176.70 & $\mathrm{H} 2 \mathrm{O}$ & -161.54 & -175.76 & $\mathrm{H}_{2} \mathrm{O}$ & -162.53 & -176.75 & $\mathrm{H}_{2} \mathrm{O}$ & -163.81 & -178.03 \\
$\mathrm{H}_{2} \mathrm{O}_{2}$ & -166.45 & -173.21 & $\mathrm{H} 2 \mathrm{O} 2$ & -165.55 & -172.31 & $\mathrm{H}_{2} \mathrm{O}_{2}$ & -166.57 & -173.33 & $\mathrm{H}_{2} \mathrm{O}_{2}$ & -170.71 & -177.47 \\
$2 \mathrm{O}$ & -155.11 & -168.63 & $2 \mathrm{O}$ & -155.11 & -168.63 & $2 \mathrm{O}$ & -154.44 & -167.96 & $2 \mathrm{O}$ & -160.88 & -174.39 \\
$\mathrm{OHO}$ & -161.30 & -171.44 & $\mathrm{OHO}$ & -158.97 & -169.11 & $\mathrm{OHO}$ & -159.87 & -170.01 & $\mathrm{OHO}$ & -165.79 & -175.93 \\
$\mathrm{H}$ & -150.16 & & $\mathrm{H}$ & -148.79 & & $\mathrm{H}$ & -149.99 & & $\mathrm{H}$ & -153.52 & \\
\hline $2 \mathrm{Ni}$ & -129.07 & -151.32 & $2 \mathrm{Pd}$ & -129.06 & -151.31 & $2 \mathrm{Pt}$ & -127.91 & -150.16 & $2 \mathrm{Ru}$ & -130.79 & -153.04 \\
\hline $\mathrm{O}_{2}$ & -139.43 & -152.95 & $\mathrm{O} 2$ & -138.26 & -151.78 & $\mathrm{O}$ & -138.12 & -151.64 & $\mathrm{O}_{2}$ & -142.17 & -155.69 \\
$\mathrm{OOH}$ & -143.19 & -153.32 & $\mathrm{OOH}$ & -141.79 & -151.93 & $\mathrm{OOH}$ & -141.63 & -151.77 & $\mathrm{OOH}$ & -146.06 & -156.20 \\
$\mathrm{O}$ & -133.16 & -154.14 & $\mathrm{O}$ & -131.11 & -152.09 & $\mathrm{O}$ & -132.09 & -153.07 & $\mathrm{O}$ & -135.45 & -156.43 \\
$\mathrm{OH}$ & -138.59 & -156.19 & $\mathrm{OH}$ & -136.97 & -154.57 & $\mathrm{OH}$ & -137.78 & -155.38 & $\mathrm{OH}$ & -141.28 & -158.88 \\
$\mathrm{H}_{2} \mathrm{O}$ & -143.83 & -158.05 & $\mathrm{H}_{2} \mathrm{O}$ & -142.67 & -156.89 & $\mathrm{H}_{2} \mathrm{O}$ & -143.46 & -157.68 & $\mathrm{H}_{2} \mathrm{O}$ & -145.18 & -159.40 \\
$\mathrm{H}_{2} \mathrm{O}_{2}$ & -147.76 & -154.52 & $\mathrm{H}_{2} \mathrm{O}_{2}$ & -146.64 & -153.40 & $\mathrm{H}_{2} \mathrm{O}_{2}$ & -147.51 & -154.27 & $\mathrm{H}_{2} \mathrm{O}_{2}$ & -149.10 & -155.86 \\
\hline & & & & & & & & & & & \\
\hline
\end{tabular}




\begin{tabular}{|c|c|c|c|c|c|c|c|c|c|c|c|}
\hline $2 \mathrm{O}$ & 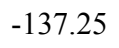 & 7 & $\mathrm{O}$ & 2 & 4 & $\mathrm{O}$ & 3 & 4 & $2 \mathrm{O}$ & 2 & -15 \\
\hline $\mathrm{HO}$ & 3. & 53 & $\mathrm{HO}$ & 40 . & 5066 & $\mathrm{HO}$ & -141.99 & 3 & $\mathrm{OHO}$ & 6 & -157 \\
\hline $\mathrm{H}$ & -131.53 & & $\mathrm{H}$ & -129 & & $\mathrm{H}$ & -131.13 & & $\mathrm{H}$ & -13 & \\
\hline $3 \mathrm{Ni}$ & -129.11 & -151. & $3 \mathrm{Pd}$ & -1 & -150.39 & $3 \mathrm{Pt}$ & -12 & & $3 \mathrm{Ru}$ & 8 & -15 \\
\hline $\mathrm{O}_{2}$ & 1 & -152 & $\mathrm{O}_{2}$ & 3 & -151 & $\mathrm{O}_{2}$ & -13 & -1 & $\mathrm{O}_{2}$ & & -15 \\
\hline $\mathrm{OOH}$ & 0 & 53 & $\mathrm{OH}$ & 1 & -151 & $\mathrm{OOH}$ & -142 & -15 & $\mathrm{OOH}$ & 8 & -156.22 \\
\hline $\mathrm{O}$ & 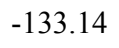 & -154 & $\mathrm{O}$ & 1 & -15 & $\mathrm{O}$ & -13 & & $\mathrm{O}$ & & - \\
\hline $\mathrm{OH}$ & 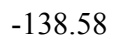 & -156 & $\mathrm{OH}$ & t & 52 & $\mathrm{OH}$ & -13 & & $\mathrm{OH}$ & & -15 \\
\hline $\mathrm{H}_{2} \mathrm{O}$ & 143 & -158 & $\mathrm{H}_{2} \mathrm{O}$ & 14 & -156 & $\mathrm{H}_{2} \mathrm{O}$ & -143 & -15 & $\mathrm{H}_{2} \mathrm{O}$ & & -15 \\
\hline $\mathrm{H}_{2} \mathrm{O}_{2}$ & דיו & -154 & $\mathrm{H}_{2} \mathrm{O}_{2}$ & 1 & -153 & $\mathrm{H}_{2} \mathrm{O}_{2}$ & -14 & 0 & $\mathrm{H}_{2} \mathrm{O}_{2}$ & & -13 \\
\hline $2 \mathrm{O}$ & 36 & -150 & $2 \mathrm{O}$ & -13 & -14 & $2 \mathrm{O}$ & -13 & -14 & $2 \mathrm{O}$ & & 110 \\
\hline $\mathrm{OHO}$ & -142.32 & -152.46 & $\mathrm{OHO}$ & -142.31 & -152 & $\mathrm{OHO}$ & -143 & -15 & $\mathrm{OHO}$ & 5 & -157 \\
\hline $\mathrm{H}$ & -131.62 & & $\mathrm{H}$ & -129 & & $\mathrm{H}$ & -13 & & $\mathrm{H}$ & & \\
\hline $4 \mathrm{Ni}$ & $02=$ & 225 & $4 \mathrm{Pd}$ & -2 & 22 & $4 \mathrm{P}$ & -20 & & $4 \mathrm{Ru}$ & 2 & -22 \\
\hline $\mathrm{O}_{2}$ & 1. & -226 & $\mathrm{O}_{2}$ & -2 & -220 & $\mathrm{O}_{2}$ & -21 & -22 & $\mathrm{O}_{2}$ & & -22 \\
\hline $\mathrm{OOH}$ & 12 & -227 & $\mathrm{OOH}$ & -215 & -22 & $\mathrm{OOH}$ & -21 & -22 & $\mathrm{OOH}$ & -2 & -23 \\
\hline $\mathrm{O}$ & -206.99 & -227 . & $\mathrm{O}$ & -205 & -226 & $\mathrm{O}$ & -20 & -22 & $\mathrm{O}$ & & -23 \\
\hline $\mathrm{OH}$ & -212.53 & -230 . & $\mathrm{OH}$ & -210 & -228.58 & $\mathrm{OH}$ & -212 & -22 & $\mathrm{OH}$ & -2 & -23 \\
\hline $\mathrm{H}_{2} \mathrm{O}$ & -217.83 & -232.05 & $\mathrm{H}_{2} \mathrm{O}$ & -216.64 & -230.86 & $\mathrm{H}_{2} \mathrm{O}$ & -217 & -23 & $\mathrm{H}_{2} \mathrm{O}$ & -2 & -23 \\
\hline $\mathrm{H}_{2} \mathrm{O}_{2}$ & -221.81 & -228.57 & $\mathrm{H}_{2} \mathrm{O}_{2}$ & -220.65 & -227.41 & $\mathrm{H}_{2} \mathrm{O}_{2}$ & -221.66 & -228.42 & $\mathrm{H}_{2} \mathrm{O}_{2}$ & -222.85 & -229.61 \\
\hline $2 \mathrm{O}$ & -211.10 & -224 & $2 \mathrm{O}$ & -210 & -224.50 & $2 \mathrm{O}$ & -210 & -22 & $2 \mathrm{O}$ & & -230.09 \\
\hline $\mathrm{OHO}$ & -216.34 & -226.48 & $\mathrm{OHO}$ & -216 & -226.21 & $\mathrm{OHO}$ & -216.50 & -226.64 & $\mathrm{OHO}$ & -221.19 & -231.33 \\
\hline $\mathrm{H}$ & -205.47 & & $\mathrm{H}$ & -205.47 & & $\mathrm{H}$ & & & $\mathrm{H}$ & -208.77 & \\
\hline
\end{tabular}

Table.S4 Gibbs free energy (in eV) of adsorbates on $\mathrm{M} @ \mathrm{Gra}(\mathrm{M}=\mathrm{Ni}, \mathrm{Pd}, \mathrm{Pt}$, and Ru) calculated by using follow formula $\left(\mathrm{x} / 2 \mathrm{O}_{2}+\mathrm{yH}^{+} \rightarrow \mathrm{H}_{\mathrm{y}} \mathrm{O}_{\mathrm{x}}\right.$ ), it defined as $\Delta G\left[{ }^{*} \mathrm{H}_{\mathrm{x}} \mathrm{O}_{\mathrm{y}}\right]=\mathrm{G}\left[{ }^{*} \mathrm{H}_{\mathrm{x}} \mathrm{O}_{\mathrm{y}}\right]-\mathrm{x} / 2 * \mathrm{G}\left[\mathrm{O}_{2}\right]-\mathrm{y} / 2 * \mathrm{G}\left[\mathrm{H}_{2}\right]$. The values in bold denote more stable intermediates of the same protonation steps along the possible reaction pathway. Values of ORR Intermediates on a single atom-doped graphene monolayer.

\begin{tabular}{ccccccccc}
\hline System & $1 \mathrm{Ni}$ & $1 \mathrm{Pd}$ & $1 \mathrm{Pt}$ & $1 \mathrm{Ru}$ & $2 \mathrm{Ni}$ & $2 \mathrm{Pd}$ & $2 \mathrm{Pt}$ & $2 \mathrm{Ru}$ \\
\hline$* \mathrm{O}_{2}$ & 1.39 & 1.13 & 1.12 & 2.78 & 1.63 & 0.47 & 1.48 & 2.65 \\
$* \mathrm{OOH}$ & 0.32 & 0.13 & 0.11 & 0.70 & 0.37 & 0.15 & 0.13 & 0.51 \\
$* \mathrm{O}$ & 0.64 & 0.17 & 0.40 & 0.07 & 0.81 & 0.16 & 1.30 & 0.23 \\
$* \mathrm{OH}$ & 2.22 & 2.56 & 2.35 & 1.92 & 2.05 & 2.48 & 2.31 & 2.45 \\
$* \mathrm{H}_{2} \mathrm{O}$ & 1.90 & 2.25 & 2.25 & 0.84 & 1.86 & 2.32 & 2.30 & 0.52 \\
$* \mathrm{H}_{2} \mathrm{O}_{2}$ & 1.28 & 1.53 & 1.18 & 2.27 & 1.20 & 1.47 & 2.50 & 0.34 \\
$* 2 \mathrm{O}$ & -1.60 & -0.89 & -2.55 & 2.68 & -0.55 & -3.27 & -1.32 & 2.09 \\
$* \mathrm{OHO}$ & 2.81 & 0.48 & 2.04 & 1.53 & 2.43 & 2.63 & 3.29 & 2.36 \\
\hline $\mathrm{System}$ & $3 \mathrm{Ni}$ & $3 \mathrm{Pd}$ & $3 \mathrm{Pt}$ & $3 \mathrm{Ru}$ & $4 \mathrm{Ni}$ & $4 \mathrm{Pd}$ & $4 \mathrm{Pt}$ & $4 \mathrm{Ru}$ \\
\hline$* \mathrm{O}_{2}$ & 1.57 & 1.36 & 1.32 & 2.65 & 1.17 & 1.15 & 1.13 & 0.86 \\
$* \mathrm{OOH}$ & 0.31 & 0.11 & 0.09 & 0.55 & 0.28 & 0.05 & 0.39 & 0.69 \\
$* \mathrm{O}$ & 0.89 & 0.18 & 1.52 & 0.19 & 0.70 & 0.82 & 0.20 & 0.84 \\
$* \mathrm{OH}$ & 2.06 & 2.58 & 1.17 & 0.62 & 2.16 & 2.04 & 2.33 & 1.13 \\
$* \mathrm{H}_{2} \mathrm{O}$ & 1.84 & 2.20 & 2.30 & 2.35 & 1.93 & 2.28 & 2.20 & 3.25 \\
$* \mathrm{H}_{2} \mathrm{O}_{2}$ & 1.27 & 1.56 & 1.63 & 0.37 & 1.30 & 1.69 & 1.31 & 0.84 \\
$* 2 \mathrm{O}$ & -1.28 & -1.05 & -2.23 & 0.34 & -1.19 & -0.12 & -1.20 & 1.20 \\
\hline
\end{tabular}




\begin{tabular}{lllllllll}
\hline$* \mathrm{OHO}$ & 2.38 & 3.11 & 4.28 & 4.13 & 1.86 & 1.72 & 2.25 & 1.23 \\
\hline
\end{tabular}

Table S5.The change of Gibbs free energy profile for the ORR to $\mathrm{H}_{2} \mathrm{O}$ or $\mathrm{H}_{2} \mathrm{O}_{2}$ on $\mathrm{M} @$ Gra at $\mathrm{U}_{0}(\mathrm{U}=0), \mathrm{U}_{\mathrm{ORR}}$ and equilibrium potential $(-1.23 \mathrm{~V})$.

\begin{tabular}{|c|c|c|c|c|c|c|c|c|c|c|c|c|}
\hline \multirow{2}{*}{$\begin{array}{c}\text { System } \\
\text { Sur } \\
\end{array}$} & \multicolumn{3}{|c|}{$1 \mathrm{Ni}$} & \multicolumn{3}{|c|}{$1 \mathrm{Pd}$} & \multicolumn{3}{|c|}{$1 \mathrm{Pt}$} & \multicolumn{3}{|c|}{$1 \mathrm{Ru}$} \\
\hline & $\mathrm{U}_{0}$ & $\mathrm{U}_{\mathrm{ORR}}$ & Ueq & $\mathrm{U}_{0}$ & $\mathrm{U}_{\mathrm{ORR}}$ & Ueq & $\mathrm{U}_{0}$ & $\mathrm{U}_{\mathrm{ORR}}$ & Ueq & $\mathrm{U}_{0}$ & $\mathrm{U}_{\mathrm{ORR}}$ & $\mathrm{U}_{\mathrm{eq}}$ \\
\hline $\mathrm{O}_{2}$ & 4.92 & 2.32 & 0.00 & 4.92 & 4.26 & 0.00 & 4.92 & 3.32 & 0.00 & 4.92 & 1.80 & 0.00 \\
\hline $\mathrm{OOH}$ & 3.23 & 1.28 & -0.46 & 3.66 & 3.17 & -0.03 & 3.68 & 2.49 & -0.01 & 2.43 & 0.10 & -1.26 \\
\hline $\mathrm{O}$ & 2.58 & 1.28 & 0.12 & 3.50 & 3.17 & 1.04 & 3.28 & 2.49 & 0.82 & 1.66 & 0.10 & -0.80 \\
\hline $\mathrm{OH}$ & 0.36 & -0.29 & -0.87 & 0.94 & 0.77 & -0.29 & 0.93 & 0.53 & -0.30 & -0.56 & -1.33 & -1.79 \\
\hline $\mathrm{H}_{2} \mathrm{O}$ & 0.00 & 0.00 & 0.00 & 0.00 & 0.00 & 0.00 & 0.00 & 0.00 & 0.00 & 0.00 & 0.00 & 0.00 \\
\hline \multicolumn{2}{|l|}{ System } & \multicolumn{2}{|l|}{$2 \mathrm{Ni}$} & \multicolumn{3}{|c|}{$2 \mathrm{Pd}$} & \multicolumn{3}{|c|}{$2 \mathrm{Pt}$} & \multicolumn{3}{|c|}{$2 \mathrm{Ru}$} \\
\hline Sur & $\mathrm{U}_{0}$ & $\mathrm{U}_{\mathrm{ORR}}$ & Ueq & $\mathrm{U}_{0}$ & $\mathrm{U}_{\mathrm{ORR}}$ & Ueq & $\mathrm{U}_{0}$ & $\mathrm{U}_{\mathrm{ORR}}$ & Ueq & $\mathrm{U}_{0}$ & $\mathrm{U}_{\mathrm{ORR}}$ & $\mathrm{U}_{\mathrm{eq}}$ \\
\hline $\mathrm{O}_{2}$ & 4.92 & 1.67 & 0.00 & 4.92 & 4.27 & 0.00 & 4.92 & 2.92 & 0.00 & 4.92 & 3.99 & 0.00 \\
\hline $\mathrm{OOH}$ & 2.91 & 0.47 & -0.78 & 4.30 & 3.81 & 0.61 & 3.31 & 1.81 & -0.38 & 1.76 & 1.06 & -1.93 \\
\hline $\mathrm{O}$ & 2.10 & 0.47 & -0.36 & 4.14 & 3.81 & 1.68 & 2.81 & 1.81 & 0.35 & 1.53 & 1.06 & -0.93 \\
\hline $\mathrm{OH}$ & 0.05 & -0.76 & -1.18 & 1.66 & 1.49 & 0.43 & 0.80 & 0.30 & -0.43 & -0.92 & -1.15 & -2.15 \\
\hline $\mathrm{H}_{2} \mathrm{O}$ & 0.00 & 0.00 & 0.00 & 0.00 & 0.00 & 0.00 & 0.00 & 0.00 & 0.00 & 0.00 & 0.00 & 0.00 \\
\hline \multicolumn{2}{|l|}{ System } & \multicolumn{2}{|l|}{$3 \mathrm{Ni}$} & \multicolumn{3}{|c|}{$3 \mathrm{Pd}$} & \multicolumn{3}{|c|}{$3 \mathrm{Pt}$} & \multicolumn{3}{|c|}{$3 \mathrm{Ru}$} \\
\hline Sur & $\mathrm{U}_{0}$ & $\mathrm{U}_{\mathrm{ORR}}$ & Ueq & $\mathrm{U}_{0}$ & $\mathrm{U}_{\mathrm{ORR}}$ & Ueq & $\mathrm{U}_{0}$ & $\mathrm{U}_{\mathrm{ORR}}$ & Ueq & $\mathrm{U}_{0}$ & $\mathrm{U}_{\mathrm{ORR}}$ & $\mathrm{U}_{\mathrm{eq}}$ \\
\hline $\mathrm{O}_{2}$ & 4.92 & 1.38 & 0.00 & 4.92 & 4.19 & 0.00 & 4.92 & 2.85 & 0.00 & 4.92 & 4.16 & 0.00 \\
\hline $\mathrm{OOH}$ & 3.04 & 0.38 & -0.65 & 3.46 & 2.91 & -0.23 & 3.51 & 1.96 & -0.18 & 1.72 & 1.15 & -1.97 \\
\hline $\mathrm{O}$ & 2.15 & 0.38 & -0.31 & 3.28 & 2.91 & 0.82 & 3.00 & 1.96 & 0.54 & 1.53 & 1.15 & -0.93 \\
\hline $\mathrm{OH}$ & 0.09 & -0.79 & -1.14 & 0.69 & 0.51 & -0.54 & 0.82 & 0.31 & -0.41 & 0.91 & 0.72 & 0.72 \\
\hline $\mathrm{H}_{2} \mathrm{O}$ & 0.00 & 0.00 & 0.00 & 0.00 & 0.00 & 0.00 & 0.00 & 0.00 & 0.00 & 0.00 & 0.00 & 0.00 \\
\hline \multicolumn{2}{|l|}{ System } & \multicolumn{2}{|l|}{$4 \mathrm{Ni}$} & \multicolumn{3}{|c|}{$4 \mathrm{Pd}$} & \multicolumn{3}{|c|}{$4 \mathrm{Pt}$} & \multicolumn{3}{|c|}{$4 \mathrm{Ru}$} \\
\hline Sur & $\mathrm{U}_{0}$ & $\mathrm{U}_{\mathrm{ORR}}$ & Ueq & $\mathrm{U}_{0}$ & $\mathrm{U}_{\mathrm{ORR}}$ & Ueq & $\mathrm{U}_{0}$ & $\mathrm{U}_{\mathrm{ORR}}$ & Ueq & $\mathrm{U}_{0}$ & $\mathrm{U}_{\mathrm{ORR}}$ & $\mathrm{U}_{\mathrm{eq}}$ \\
\hline $\mathrm{O}_{2}$ & 4.92 & 2.12 & 0.00 & 4.92 & 1.65 & 0.00 & 4.92 & 4.13 & 0.00 & 4.92 & 1.56 & 0.00 \\
\hline $\mathrm{OOH}$ & 3.46 & 1.37 & -0.23 & 3.82 & 1.36 & 0.13 & 3.40 & 2.80 & -0.29 & 3.37 & 0.84 & -0.32 \\
\hline $\mathrm{O}$ & 2.76 & 1.37 & 0.30 & 3.00 & 1.36 & 0.54 & 3.20 & 2.80 & 0.74 & 2.52 & 0.84 & 0.06 \\
\hline $\mathrm{OH}$ & 0.61 & -0.09 & -0.62 & 0.96 & 0.14 & 0.81 & 0.87 & 0.67 & -0.36 & 1.40 & 0.56 & 0.17 \\
\hline $\mathrm{H}_{2} \mathrm{O}$ & 0.00 & 0.00 & 0.00 & 0.00 & 0.00 & 0.00 & 0.00 & 0.00 & 0.00 & 0.00 & 0.00 & 0.00 \\
\hline
\end{tabular}



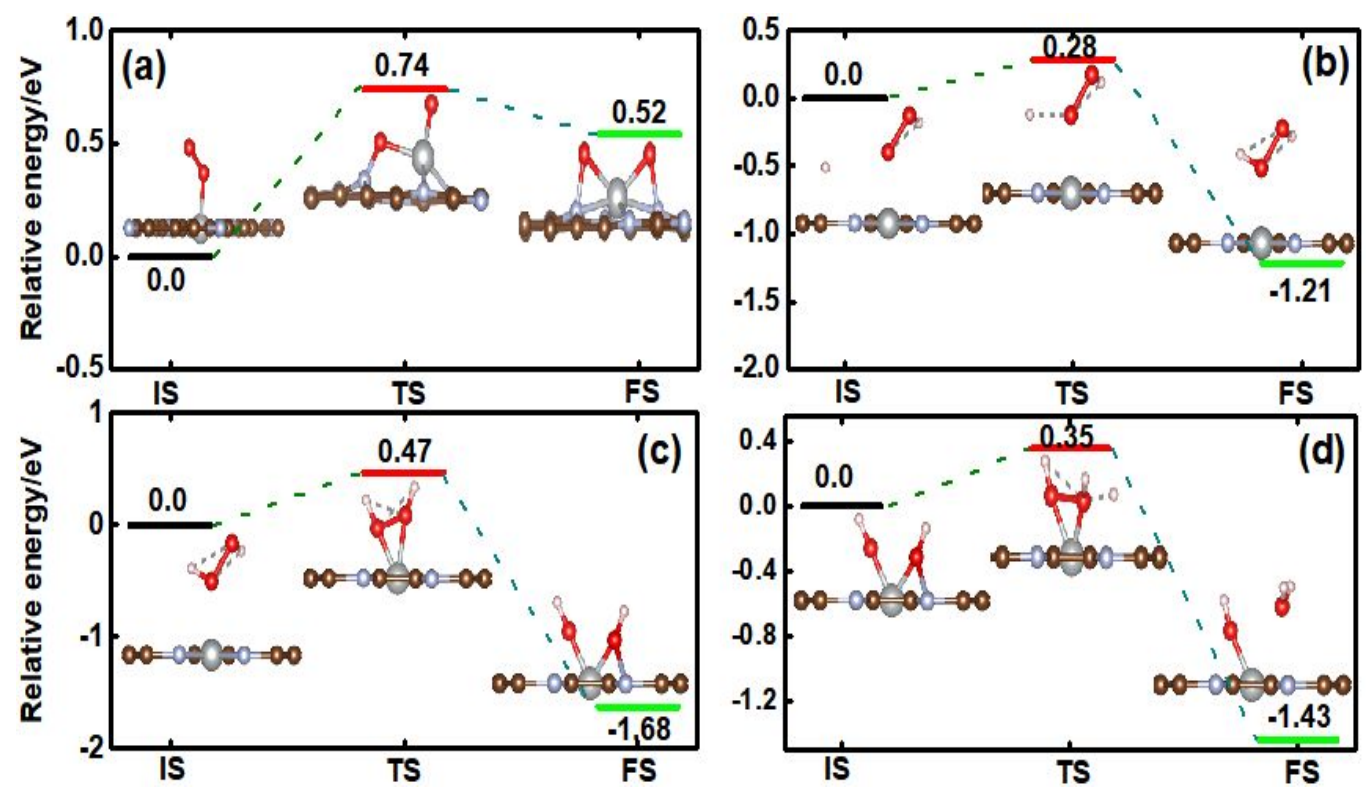

Figure S5. Optimized geometric structures of the initial (IS), transition (TS), and final (FS) states of ORR elemental steps on 2Ni@Gra surface and the corresponding activation energy for the reaction: $(\mathrm{a}) * \mathrm{O}_{2} \rightarrow{ }^{*} \mathrm{O}+* \mathrm{O}$;

(b) $* \mathrm{OOH}+\mathrm{H}^{+}+\mathrm{e}^{-} \rightarrow * \mathrm{H}_{2} \mathrm{O}_{2}$ (c)* $\mathrm{OOH}+\mathrm{H}^{+}+\mathrm{e}^{-} \rightarrow 2 * \mathrm{OH}$, and $2 * \mathrm{OH}+\mathrm{H}^{+}+\mathrm{e}^{-} \rightarrow * \mathrm{OH}+\mathrm{H}_{2} \mathrm{O}$, respectively.
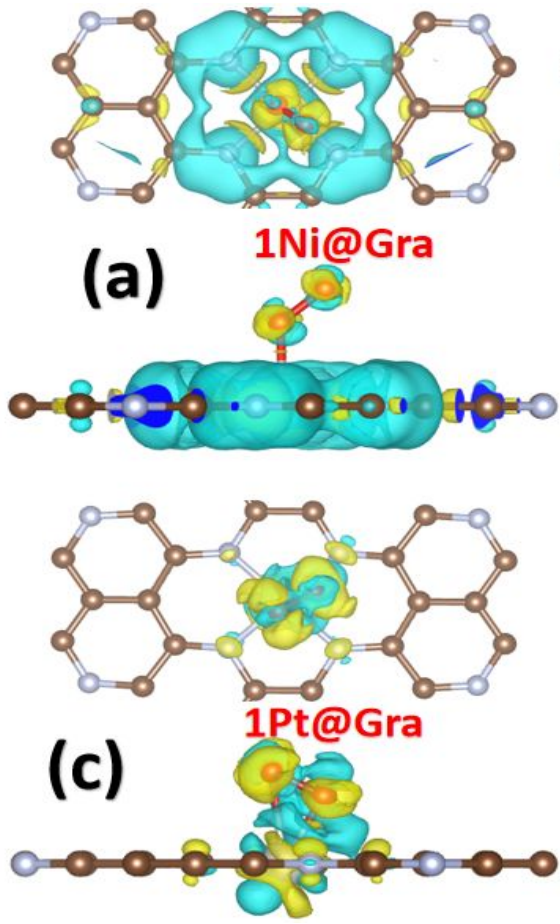
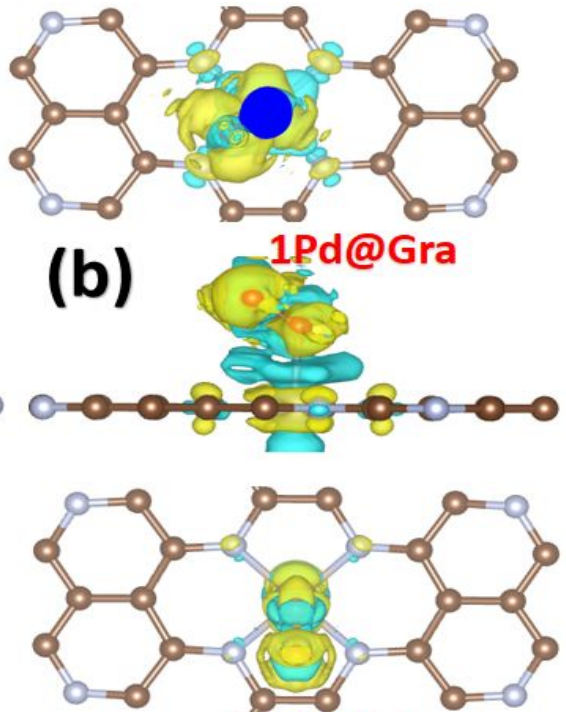

(d)
1Ru@Gra

Figure.S6 The differential charge density distribution for $\mathrm{M} @ \mathrm{SV}$ obtained via $\rho$ diff $=\rho$ tot $-\rho \mathrm{O}_{2}-\rho$ Sur represents the charge redistribution upon formation of 1M@Gra (M=Ni, Pd, Pt and Rh, (a) 1Ni@Gra, (b) 1Pd@Gra, (c) 1Pt@Gra, and (d)1Ru@Gra, respectively.

\section{References}

[1] Calle-Vallejo F, Martinez J I, Rossmeisl J. Density functional studies of functionalized graphitic materials with late transition metals for oxygen reduction reactions[J]. Physical Chemistry Chemical Physics, 2011, 13(34):

15639-15643.

[2] Li N, Chen X, Ong W J, et al. Understanding of electrochemical mechanisms for $\mathrm{CO}_{2}$ capture and conversion into hydrocarbon fuels in transition-metal carbides (MXenes) [J]. ACS nano, 2017, 11(11): 10825-10833.

[3] Abild-Pedersen F, Greeley J, Studt F, et al. Scaling properties of adsorption energies for hydrogen-containing molecules on transition-metal surfaces[J]. Physical review letters, 2007, 99(1): 016105. 
[4] Vanderbilt D. Soft self-consistent pseudopotentials in a generalized eigenvalue formalism[J]. Physical review B, 1990, 41(11): 7892.

[5] Atkins, P. W. Physical Chemistry, 6th ed., Oxford University Press: Oxford, U.K., 1998; pp 485, 925-927, 942. 